\title{
Escala BISAP en pancreatitis aguda. Azogues 2016-2017. Estudio descriptivo.
}

BISAP scale in acute pancreatitis. Azogues 2016-2017. Descriptive study.

Berrezueta Córdova Diego Andrés', Jácome Morejón Pablo Andrés

\section{RESUMEN}

Objetivo: aplicar la escala Bedside Index for Severity in Acute Pancreatitis

VOLUMEN 37 | Nº 2 I SEPTIEMBRE 2019

FECHA DE RECEPCIÓN: 12/06/2019 FECHA DE APROBACIÓN: 17/07/2019 FECHA PUBLICACIÓN: 10/09/2019

\section{Libre ejercicio}

DOI: https://doi.org/10.18537/RFCM.37.02.03

Correspondencia:

diego.berrezuetaQucuenca.edu.ec

Dirección:

Ciudadela Capulies, Ochoa León

Código Postal:

010113

Celular:

0995011709

Cuenca - Ecuador (BISAP) a los expedientes clínicos de pacientes con diagnóstico de pancreatitis aguda en el hospital Homero Castanier Crespo, Azogues enero de 2016 a diciembre de 2017.

Metodología: se realizó un estudio cuantitativo, descriptivo y retrospectivo, se trabajó con el universo de expedientes clínicos con reporte de pancreatitis aguda de 2016 a 2017. Los datos se analizaron con los programas SPSS versión 20 y Excel, utilizando distribuciones de frecuencia y porcentajes.

Resultados: se estudiaron 79 casos, encontrando una edad media de 48.5 años; el $57 \%$ correspondió al sexo femenino. Se obtuvo una mortalidad en general del $12.7 \%$; siendo la forma leve el $79.7 \%$ de los casos sin fallecimientos; contrastando con la forma grave con el $20.3 \%$, de los cuales el $62.5 \%$ fallecieron.

Conclusiones: BISAP demostró ser un predictor de mortalidad, accesible, rápido y útil. Se aprecia una tendencia al incremento de la mortalidad conforme se eleva el puntaje de la escala, siendo esta directamente proporcional a la aparición de complicaciones locales y sistémicas propias de la enfermedad.

Palabras Clave: pancreatitis, indicadores de morbimortalidad, pronostico, BISAP. 


\section{ABSTRACT}

Objective: to apply the Bedside Index for Severity in Acute Pancreatitis (BISAP) scale to the clinical records of patients diagnosed with acute pancreatitis at the Hospital Homero Castanier Crespo, Azogues January 2016 to December 2017.

Methodology: a quantitative, descriptive and retrospective study was carried out; the universe of clinical records with reports of acute pancreatitis was worked from 2016 to 2017 . The data were analyzed with the SPSS version 20 and Excel programs, using frequency distributions and percentages.

Results: a total of 79 cases were studied, finding an average age of 48.5 years; the $57 \%$ corresponded to the female sex. A general mortality of $12.7 \%$ was obtained; being the mild form $79.7 \%$ of the cases without deaths; and contrasting with the serious form with $20.3 \%$, of which $62.5 \%$ died.

Conclusions: BISAP proved to be a mortality predictor; it is accessible, fast and useful. A tendency to increase mortality at the same time in the score of the escalation was observed, being this directly proportional to the incidence of local and systemic complications of the disease.

Keywords: pancreatitis, indicators of morbidity and mortality, prognosis, BISAP.

\section{INTRODUCCIÓN}

La pancreatitis aguda es un proceso inflamatorio rápidamente desarrollado del páncreas que varía en términos de presentación clínica y gravedad [1]. Aunque la mayoría de los casos son leves y el pronóstico es bueno, todavía hay entre el 20 y el $30 \%$ de los pacientes que presentan complicaciones graves [2]. Actualmente la mortalidad general debida a pancreatitis aguda es del $4 \%$ al $10 \%$, sin embargo, en casos graves puede llegar al $30 \%$ [3].

La incidencia de pancreatitis aguda varía entre 30 a 50 por 100,000 habitantes [4], la incidencia mundial está aumentando, lo que aumenta aún más su carga sobre los servicios de salud [5,6]. En Ecuador se registraron 4,374 egresos en el año 2014, con una tasa de letalidad de 2.47 por cada 100 casos [7]
Con más de 275,000 hospitalizaciones anuales en Estados Unidos, la pancreatitis aguda es la tercera causa más común de afecciones gastrointestinales que requieren hospitalización aguda [5,6]. En Ecuador, si bien no existen cifras oficiales en cuanto a inversión económica, según el INEC [7] se tiene un promedio de 8 días de hospitalización que representan altos costos tanto para el país como para la familia.

La pancreatitis aguda es una enfermedad difícil, al no comprender completamente su desarrollo y no existe una intervención específica para prevenir o tratar la aparición y progresión de la enfermedad [8]. Puede deteriorar significativamente la calidad de vida de los pacientes incluso pasado un año luego del episodio y además con recaídas frecuentes. Repercutiendo significativamente en el núcleo familiar, a esto se le suma el hecho de que afecta de manera importante a los ingresos económicos al determinar una disminución de las actividades laborales del paciente.

La pancreatitis aguda leve se caracteriza por la ausencia de falla orgánica y de complicaciones locales o sistémicas. Estos pacientes generalmente ingresan durante la fase temprana, el tratamiento sobre todo es de apoyo, no requieren estudios de imagen pancreática y su mortalidad es muy rara [9].

La pancreatitis aguda grave se caracteriza por la presencia de falla orgánica o de complicaciones locales o sistémicas [9]. Schepers et al. [10] en su estudio retrospectivo indica que la mortalidad es mayor en los pacientes con insuficiencia orgánica sola $(44 \%)$ que en los pacientes con insuficiencia orgánica y necrosis pancreática infectada $(29 \%)$, pero la insuficiencia orgánica persistente también suele desarrollar complicaciones locales.

El objetivo del estudio es aplicar la escala BISAP como predictor de mortalidad, para que a futuro de ser posible convertirla en una escala dentro del protocolo de manejo pancreatitis aguda, utilizandola en la estadificación de los pacientes para poder realizar un manejo terapéutico adecuado, disminuir la morbimortalidad y administrar correctamente el uso de recursos hospitalarios.

En los hospitales de nuestro medio no se cuenta con la tecnología y recursos necesarios para dar un pronóstico adecuado del paciente con pancrea- 
titis aguda por lo que se ha propuesto la posibilidad de implementar el score BISAP que no requiere exámenes adicionales a los hechos de rutina y que posee una sensibilidad de $61.11 \%$ y especificidad de $96.33 \%$ [11].

Zhou et al., [1] en su estudio en el hospital de Chao-yang Beijing del 2014 a 2017; los valores del área debajo de la curva de: relación neutrófilo-linfocito (NLR), proporción de plaquetas-linfocitos (PLR), amplitud de distribución eritrocitaria (RDW), nitrógeno ureico en sangre (BUN) y scores SOFA, BISAP, Ranson y APACHE II para predecir pancreatitis aguda severa fueron $0.722,0.621,0.787$, $0.677,0.806,0.841,0.806$ y 0.752 , respectivamente; mientras que sus valores del área debajo de la curva para predecir la mortalidad a los 28 días fue de $0.851,0.693,0.885,0.765,0.968,0.929,0.812$ y 0.867 , respectivamente.

BISAP logró el área debajo de la curva, la sensibilidad y el valor de predicción negativo más altos para predecir pancreatitis aguda severa, mientras que SOFA presentó una leve superioridad para predecir la mortalidad. Ambas fueron factores de riesgo independientes para predecir pancreatitis aguda severa, pero BISAP presentó una relación más fuerte que SOFA, odds ratio de 2.145 vs 1.554 [1].

BISAP estratifica en dos grupos: leve (puntaje $<3$ ) y grave (puntaje $\geq 3$ ); 1 punto por cada parámetro presente:

\title{
Cuadro $N^{\circ} 1$
}

Parámetros del score BISAP

\author{
BUN >25 mg/dl ( $8.9 \mathrm{mmol} / \mathrm{L})$. \\ Estado mental anormal con una puntuación de coma en Glasgow $<15$. \\ Síndrome de respuesta inflamatoria sistémica (SRIS)* \\ Edad del paciente $>60$ años. \\ Estudio de imagen que revela derrame pleural.
}

*SRIS: presencia de 2 o más de los siguientes criterios: temperatura $<36^{\circ} \mathrm{C}$ o $>38^{\circ} \mathrm{C}$; respiraciones $>20 / \mathrm{min}$ o PaCO2 $<32 \mathrm{mmHg}$; frecuencia cardíaca $>90 / \mathrm{min}$; leucocitos $<4.000 / \mathrm{mm} 3$ o $>12.000 / \mathrm{mm} 3$ o $>10 \%$ de formas inmaduras encontradas en el frotis de sangre.

Fuente: Fisher et al. Schwartz principios de cirugía. 2015 [12]

\section{METODOLOGÍA}

Se diseñó un estudio cuantitativo, descriptivo y retrospectivo; se trabajó con el universo, el mismo que estuvo conformado de 79 expedientes clínicos de pacientes con diagnóstico de pancreatitis aguda durante los años 2016 y 2017 en el hospital Homero Castanier Crespo. Se excluyó los expedientes clínicos de pacientes con diagnóstico no confirmado de pancreatitis aguda, de igual manera en los que la información se encontraba incompleta y aquellos en los que la fecha de ingreso o egreso se encontraba fuera del tiempo de estudio.

Se recolectaron los datos en un formulario elaborado por los autores diseñado para el estudio previamente revisado por la Comisión de Pro- yectos de la Facultad de Ciencias Médicas de la Universidad de Cuenca, y se procesaron los datos en los programas SPSS 20 y Excel, mediante frecuencias, porcentajes y medidas de tendencia central. Las variables utilizadas fueron: grupo etario, sexo, score BISAP: nitrógeno ureico en sangre, estado de conciencia, presencia de derrame pleural, edad y síndrome de respuesta inflamatoria sistémica; y condición de egreso. 


\section{RESULTADOS}

Tabla $N^{\circ} 1$

Distribución según sexo y grupos etarios.

\begin{tabular}{lcc}
\hline $\begin{array}{l}\text { Variable } \\
\text { Sexo }\end{array}$ & $\mathbf{n}$ & $\%$ \\
Hombre & 34 & 43 \\
Mujer & 45 & 57 \\
Grupo etario (años) & & \\
$\leq 20$ & 4 & 5.1 \\
21 a 34 años & 28 & 35.4 \\
35 a 49 años & 14 & 17.7 \\
50 a 64 años & 9 & 11.4 \\
mayores de 65 años & 24 & 30.4 \\
Total & 79 & 100 \\
\hline
\end{tabular}

Fuente: expedientes clínicos

Elaborado por: autores

De 79 pacientes estudiados, se encontró un rango de edad de 16 a 94 años, con una media de edad del grupo de estudio de 48.5 años con una DS de 22.5 .

\section{Tabla $\mathrm{N}^{\circ} 2$}

Distribución de los pacientes según los parámetros de BISAP.

\begin{tabular}{|c|c|c|}
\hline Variable & $\mathbf{n}$ & $\%$ \\
\hline \multicolumn{3}{|c|}{ BUN >25 mg/dl } \\
\hline $\mathrm{Si}$ & 23 & 29.1 \\
\hline No & 56 & 70.9 \\
\hline \multicolumn{3}{|c|}{ Glasgow $<15$ puntos } \\
\hline Si & 8 & 10.1 \\
\hline No & 71 & 89.9 \\
\hline \multicolumn{3}{|c|}{ Edad $>60$ años } \\
\hline Si & 24 & 30.4 \\
\hline No & 55 & 69.6 \\
\hline \multicolumn{3}{|c|}{ Derrame pleural } \\
\hline $\mathrm{Si}$ & 22 & 27.8 \\
\hline No & 57 & 72.2 \\
\hline \multicolumn{3}{|c|}{ Síndrome de respuesta inflamatoria sistémica } \\
\hline $\mathrm{Si}$ & 54 & 68.4 \\
\hline No & 25 & 31.6 \\
\hline Total & 79 & 100 \\
\hline
\end{tabular}

Fuente: expedientes clínicos

Realizado por: autores 


\section{Tabla $N^{\circ} 3$}

Distribución según la gravedad de pancreatitis aguda.

\begin{tabular}{lcc}
\hline Variable & $\mathbf{n}$ & $\%$ \\
Gravedad de la pancreatitis aguda & & \\
$\quad$ Leve & 63 & 79.7 \\
$\quad$ Grave & 16 & 20.3 \\
\hline Total & 79 & 100 \\
\hline
\end{tabular}

Fuente: expedientes clínicos

Realizado por: autores

Tabla $N^{\circ} 4$

Distribución de acuerdo a la gravedad y la condición de egreso de los pacientes.

\begin{tabular}{ccccc}
\hline Variable & \multicolumn{4}{c}{ Condición de egreso } \\
\hline Gravedad de la pancreatitis aguda & \multicolumn{2}{c}{ Vive } & Muere \\
& $\mathbf{n}$ & $\%$ & $\mathbf{n}$ & $\%$ \\
\hline Casos leves & 63 & 100 & 0 & 0 \\
0 puntos & 12 & 100 & 0 & 0 \\
1 punto & 28 & 100 & 0 & 0 \\
2 puntos & 23 & 100 & 0 & 0 \\
\hline Casos graves & 6 & 37.5 & 10 & 62.5 \\
3 puntos & 6 & 54.5 & 5 & 45.5 \\
4 puntos & 0 & 0 & 4 & 100 \\
5 puntos & 0 & 0 & 1 & 100 \\
\hline Total & 69 & 87.3 & 10 & 12.7 \\
\hline
\end{tabular}

Fuente: expedientes clínicos

Se evidencia en los 63 casos de pancreatitis aguda leve que el $100 \%$ sobrevivió. En cuanto a los casos graves de pancreatitis aguda, en el puntaje 3 se observa que fallecieron el $45.5 \%$; mientras que con puntaje 4 y 5 mueren el $100 \%$. Del total de casos graves el $62.5 \%$ (10 casos) fallecieron. De forma general, la mortalidad fue del $12.7 \%$ del total de casos estudiados.

\section{DISCUSIÓN}

Exponiendo el presente estudio se encontró que el sexo femenino es el más prevalente con un $57 \%$; concordando con el estudio realizado en el departamento de gastroenterología del hospital de Gra-
Realizado por: autores

nada-España en el año 2017, el cual nos muestra una prevalencia del sexo femenino de $51.1 \%$ [13]. Probablemente la diferencia entre géneros se relacione más con la causa; ya que en los varones es más frecuente que la causa sea el consumo de alcohol, en las mujeres los cálculos biliares [12].

La edad estudiada osciló entre 16 a 94 años, siendo la media de 48.5 años con un desvió estándar de \pm 22.5 años, el grupo etario más frecuente fue de 21 a 34 años con el $35.4 \%$. Anikhindi et al., [14] en el año 2015 en el instituto hindú del norte, obtuvieron en su estudio una edad media de presentación de 42 años con un rango de 12 a 90 años. La variación de la edad es atribuible a las distintas causas; ya que en la tercera y cuarta década de la vida se presenta la inducida por alcohol y fárma- 
cos, mientras que la causada por traumatismo o cálculos biliares se presenta en la sexta década de la vida [12].

Con respecto a las variables del score BISAP, se presentó SRIS en el $68.4 \%$ de casos, $30.4 \%$ tienen más de 60 años, BUN mayor de $20 \mathrm{mg} / \mathrm{dl}$ con $29.1 \%$, seguido de derrame pleural con el $27.8 \%$ y el $10.1 \%$ presento alteración del estado de conciencia. En 2017, se analizó retrospectivamente la documentación de 963 pacientes en Polonia, demostrando que los pacientes con edad $\geq 65$ años representaron el $32.5 \%$ de todos los pacientes con pancreatitis aguda [15]. Márta et al. [16] indican que los sistemas de puntuación consideran la edad avanzada como un factor de riesgo de mortalidad $(p=0.022)$ y severidad $(p<0.001)$; la elevación de 9 veces la mortalidad por encima de los 59 años sugiere la participación de factores de deterioro adicionales, como la comorbilidad en personas de edad avanzada.

La respuesta inflamatoria sistémica provocada por la tormenta de citoquinas que resulta de la lesión de células acinares en pancreatitis aguda, causa modificaciones en las variables fisiológicas lo cual repercute en los parámetros bioquímicos; SRIS se recomienda como herramienta para valorar la respuesta al tratamiento, debido que atenuarlo conducirá a menores tasas de insuficiencia orgánica y mortalidad en la pancreatitis aguda [4]. Arnedillo et al., [17] en 2017 en su trabajo sobre afectación pleuropulmonar en enfermedades del aparato digestivo, la prevalencia del derrame pleural en la pancreatitis aguda es del 4 al $20 \%$. Se explica por su patogenia relacionada con la vía linfática transdiafragmática; la inflamación del páncreas aumenta la permeabilidad de los vasos linfáticos, lo que ocasiona una obstrucción parcial o completa de esos vasos en el espacio pleural y favorece la acumulación de más líquido.

Un aumento en el nivel de BUN refleja el estado de la enfermedad de agotamiento del volumen intravascular inicial y azoemia prerrenal en pancreatitis aguda. Un estudio de validación internacional observó que un nivel de BUN de $20 \mathrm{mg} /$ dl o más al ingreso se asoció con un aumento de la incidencia de mortalidad (OR 4.6) [18]. Tiene una relación con la gravedad de la pancreatitis $(p=0.001)$ [2], lo que representa una herramienta adicional para estratificar a los pacientes con riesgo de severidad [18].
De esta manera se muestra al BISAP como una escala sencilla, rápida y accesible en la emergencia (dentro de las primeras 24 horas [2]) y brinda una visión global de la condición del paciente; que aplicado tempranamente identifica pacientes con cuadro clínico severo.

En el presente estudio la forma leve corresponde al $79.7 \%$ y la grave se presentó con un $20.3 \%$ de los casos. Del total de casos que ingresaron con diagnóstico de pancreatitis aguda, el $87.3 \%$ egreso en la condición de vivo y los casos leves clasificados mediante el score BISAP ninguno falleció, mientras que de casos graves fallecieron el $62.5 \%$. Del puntaje $3,45.5 \%$ murieron y dentro de los 2 últimos puntajes el $100 \%$ fallecieron. De forma general, la mortalidad alcanzo el $12.7 \%$. La baja mortalidad puede deberse al hecho de que más de la mitad $(79.7 \%)$ de los pacientes tenían la forma leve.

Senapati et al. [19] en el departamento de cirugía general SCB American Collegue en Odisha-India en 2014, se observó que el puntaje BISAP mayor a 3 se asoció con una tasa de mortalidad de $18 \%$ y 6.4 veces mayor probabilidad de desarrollar falla multiorgánica; los pacientes con 2 o menos puntos, el número de muertes fue menor al $1 \%$. Datos que concuerdan con el presente y otros estudios, demostrando que a mayor puntaje de BISAP mayor es la mortalidad.

La muerte temprana generalmente ocurre como resultado del síndrome de disfunción de múltiples órganos debido al SRIS las primeras 2 semanas, mientras que aproximadamente la mitad de los pacientes mueren 2 semanas después debido a necrosis peri pancreática, infección y síndrome de disfunción de múltiples órganos secundario [20]. Es importante recalcar que afecciones crónicas previas, están relacionadas con la presencia y la persistencia de la insuficiencia orgánica. Se debe prestar atención especial a los pacientes que desarrollan insuficiencia orgánica y BISAP $\geq 3$, ya que esto puede ser una señal importante de insuficiencia orgánica persistente [13] e ingreso a la unidad de cuidados intensivos [21].

Las directrices japonesas revisadas de 2015 para el tratamiento de la pancreatitis aguda recomiendan específicamente el uso de un sistema de puntuación para la evaluación de la gravedad en la práctica clínica habitual [13], encontramos que el puntaje BISAP es una herramienta importante y factible en la admisión para predecir pancreati- 
tis aguda severa y la mortalidad. Esto sugiere que más de la mitad de los pacientes con BISAP $\geq 3$ morirán. Como se observó BISAP fue superior o igual a otros predictores, siendo su virtud el disminuir el uso de recursos.

Se presentaron como limitaciones en este estudio: en primer lugar, la baja prevalencia de casos de pancreatitis aguda en comparación con otros estudios; no hemos comparado con otros predictores como lo hacen otros estudios por la falta de datos para completar los scores por lo que no conocemos su eficacia en nuestro universo.

\section{CONCLUSIONES}

BISAP demostró ser una escala predictora de mortalidad y severidad, accesible, rápida y útil. Se aprecia una tendencia al incremento de la mortalidad conforme se eleva el puntaje de la escala, siendo esta directamente proporcional a la aparición de complicaciones locales y sistémicas propias de la enfermedad.

\section{ASPECTOS BIOÉTICOS}

Previa revisión y aprobación por el comité de bioética de la Universidad de Cuenca, y con posterior autorización de la directora y jefa de estadística del hospital Homero Castanier, se trabajó a través de formularios de manera que los datos recogidos se mantuvieron en absoluta confidencialidad, la identidad de los pacientes en ningún momento fue revelada y su uso fue exclusivamente en función de este estudio.

\section{INFORMACIÓN DE LOS AUTORES}

- Berrezueta Córdova Diego Andrés. Médico. Libre Ejercicio. Cuenca - Azuay - Ecuador. e-mail: diego.berrezueta@ucuenca.edu.ec ORCID: https://orcid.org/0000-0002-7370-6907

- Jácome Morejón Pablo Andrés. Médico. Libre Ejercicio. Cuenca - Azuay - Ecuador. e-mail: andres.jacome94@ucuenca.edu.ec ORCID: https://orcid.org/0000-0001-5601-3984

\section{CONTRIBUCIÓN DE LOS AUTORES}

Los autores declaran haber contribuido de forma similar en el diseño, levantamiento, tabulación y procesamiento de la información; elaboración de marco teórico, discusión y conclusiones. Revisión bibliográfica. Elaboración y revisión final.

\section{CONFLICTO DE INTERESES}

Los autores declaran no tener conflicto de intereses en la presente investigación.

\section{FUENTES DE FINANCIAMIENTO}

Autofinanciado

\section{REFERENCIAS BIBLIOGRÁFICAS}

1. Zhou H, Mei X, He X, Lan T, Guo S. Severity stratification and prognostic prediction of patients with acute pancreatitis at early phase: A retrospective study. Medicine. 2019; 98(16): e15275. Disponible en: https://www. ncbi.nlm.nih.gov/pubmed/?term=Severity+stratification+and+prognostic+prediction+o$f+$ patients+with+acute+pancreatitis+at+early+phase $\% 3 A+A+$ retrospective+study.

2. Liu G, Tao J, Zhu Z, Wang W. The early prognostic value of inflammatory markers in patients with acute pancreatitis. Clinics and Research in Hepatology and Gastroenterology. 2018; 1-8. Disponible en: https://doi. org/10.1016/j.clinre.2018.11.002

3. Berkowitz R, Rose G. Pancreas. En: Walls Ron. Rosen's emergency medicine: concepts and clinical practice. 9 ed. Philadelphia, USA. Elsevier; 2018; 81, 1104-1111.

4. Sreejith V, Pooja G, Ujjwal S, Bhaskar T, Vishnubhatla S, Anoop S. Comparison of various scoring systems and biochemical markers in predicting the outcome in acute pancreatitis. Páncreas. 2017; 47(1): 1-7. Disponible en: https://www.ncbi.nlm.nih.gov/pubmed/?ter$\mathrm{m}=$ Comparison+of+Various+Scoring+Systems+and+Biochemical+Markers+in+Predicting+the+Outcome+in+Acute+Pancreatitis .

5. Van Dijk S, Hallensleben N, van Santvoort H, Fockens P,van Goor H, Bruno M et al. Acute pancreatitis: recent advances through randomised trials. Gut. 2017; 66: 2024-2032. Disponible en: https://www.ncbi.nlm.nih.gov/ pubmed/28838972

6. Peery A, Crockett S, Murphy C, Lund J, Dellon $\mathrm{E}$, Williams $\mathrm{J}$, et al. Burden and cost of gastrointestinal, liver, and pancreatic diseas- 
es in the United States: Update 2018. Gastroenterology. 2019; 156(1): 254-272.e11. Disponible en: https://www.ncbi.nlm.nih.gov/ pubmed/30315778

7. Instituto ecuatoriano de estadísticas y censos (INEC). Anuario camas egresos hospitalarios. 2014: 236; Disponible en: http:// www.ecuadorencifras.gob.ec/documentos/ web-inec/Estadisticas_Sociales/Camas_ Egresos_Hospitalarios/Publicaciones-Cam_ Egre_Host/Anuario_Camas_Egresos_Hospitalarios_2014.pdf

8. Sahin M, Hegyi P. Smoking and drinking synergize in pancreatitis: Multiple hits on multiple targets. Gastroenterology. 2017; 153(6): 1479-1481. Disponible en: https://www.ncbi. nlm.nih.gov/pubmed/?term=Smoking+and+drinking+synergize+in+pancreatitis $\% 3 \mathrm{~A}+-$ multiple+hits+on+multiple+target

9. Cerda J. Análisis de las nuevas clasificaciones de la pancreatitis aguda. 2013; 3(35). Disponible en: http://www.medigraphic.com/ pdfs/cirgen/cg-2013/cgs131h.pdf

10. Schepers N, Bakker O, Besselink M, Ahmed $\mathrm{U}$, Bollen T, Gooszen H et al. Impact of characteristics of organ failure and infected necrosis on mortality in necrotising pancreatitis. Gut. 2018; 0:1-8. Disponible en: https://www. ncbi.nlm.nih.gov/pubmed/29950344

11. Gaibor C, Molina J, Tenezaca Á. Eficacia de la escala BISAP para predicción temprana de severidad en pancreatitis aguda biliar. Rev HJCA. 2016; 8(2). Disponible en: http:// revistamedicahjca.med.ec/ojs/index.php/RevHJCA/article/view/143

12. Ficher W, Andersen D, Windsor J, Soluja A, Brunicardi C. Páncreas. En: Brunicardi Charles. Schwartz principios de cirugía. 10 ed. México. McGraw-Hill; 2015, 1351-1355.

13. Valverde $F$, Matas $A$, Alegría $C$, Jiménez $R$, Úbeda $M$, Redondo $E$. BISAP, RANSON, lactate and others biomarkers in prediction of severe acute pancreatitis in a European cohort. Journal of Gastroenterology and Hepatology. 2017; 32(9); 1649-1656. Disponible en: https://www.ncbi.nlm.nih.gov/ pubmed/28207167
14. Anikhindi S, Kumar A, Singla V, Sharma P, Bansal N, Arora A. Bedside Index for Severity in Acute Pancreatitis (BISAP) score best predicts 120-day mortality in patients with acute pancreatitis: A study on 358 patients from a North Indian Center. Clinical Gastroenterology and Hepatology. 2015; 13(7); e100. Disponible en: doi:10.1016/j.cgh.2015.04.103

15. Koziel D, Gluszek S. Age an independent risk factor for severe acute pancreatitis. Pancreatology. 2017; 17(3); s14. Disponible en: https://www-clinicalkey-es. pbidi.unam.mx:2443/\#!/content/journal/1-s2.0-S1424390317301382

16. Márta K, Lazarescu A, Farkas N, Mátrai P, Cazacu I, Ottóffy M et al. Aging and comorbidities in acute pancreatitis I: A meta-analysis and systematic review based on 194,702 patients. Frontiers in Physiology. 2019; 10(328). Disponible en: https://www.ncbi.nlm.nih. gov/pubmed/?term=Aging +and+Comorbidities+in+Acute+Pancreatitis $+1 \% 3 \mathrm{~A}+\mathrm{A}+\mathrm{Me}-$ ta-Analysis+and+Systematic+Review+Based+on+194\%2C702+Patients.+Frontiers+in+Physiology

17. Arnedillo A, Merino M. Afectación pleuropulmonar en las enfermedades del aparato digestivo. En: Alvarez-Sala Walther José. Neumología clínica. 2 ed. España: Elsevier; 2017. 570-578.

18. Hong W, Lin H, Zippi M, Geng W, Stock S, Zimmer V, et al. High-density lipoprotein cholesterol, blood urea nitrogen, and serum creatinine can predict severe acute pancreatitis. Biomed Res Int. 2017:1-7. Disponible en: https://doi.org/10.1155/2017/1648385

19. Senapati D, Kumar P, Sekhar S, Kumar A, Gowda M, Nath N. A prospective study of the Bedsid Index for Severity in Acute Pancreatitis (BISAP) score in acute pancreatitis: An Indian perspective. Pancreatology. 2014; 14(5); 335-339. Disponible en: http://dx.doi. org/10.1016/j.pan.2014.07.007

20. Gray R, Cagliani J, Amodu L, Nauka P, Villacres B, Santos T et al. Maximizing the use of scoring systems in the prediction of outcomes in acute pancreatitis. Digestion. 2018; 1-6. Disponible en: https://www.ncbi.nlm.nih. gov/pubmed/?term=Maximizing+the+Use+o$\mathrm{f}+$ Scoring + Systems $+\mathrm{in}+$ the + Prediction $+\mathrm{o}-$ $\mathrm{f}+$ Outcomes+in+Acute+Pancreatitis 
21. Tee $\mathrm{Y}$, Fang $\mathrm{H}$, Kuo $\mathrm{I}$, Lin $\mathrm{Y}$, Huang $\mathrm{S}$, $\mathrm{Yu}$ $M$. Serial evaluation of the SOFA score is reliable for predicting mortality in acute severe pancreatitis. Medicine. 2018;97(7): 1-6.

Disponible en: http://dx.doi.org/10.1097/ MD.0000000000009654 